\title{
Measurement of Boer-Mulders Function via Drell-Yan Process by SeaQuest Experiment at Fermilab
}

\author{
Kenichi Nakano \\ (for the E906/SeaQuest Collaboration) \\ Physics Department, Tokyo Institute of Technology \\ 2-12-1-H-31, Ookayama, Meguro-ku, Tokyo 152-8550, Japan \\ knakano@nucl.phys.titech.ac.jp \\ Published 29 February 2016
}

\begin{abstract}
The SeaQuest experiment is being carried out at Fermi National Accelerator Lab (FNAL) to investigate the nucleon structure with the Drell-Yan process. It utilizes the $120-\mathrm{GeV}$ proton beam extracted from the FNAL Main Injector and targets of liquid hydrogen, liquid deuterium, carbon, iron and tungsten. The solid targets are used to measure the nuclear effects. This paper describes the flavor asymmetry of light anti-quark distributions in the proton $(\bar{d} / \bar{u})$ and the angular distribution of Drell-Yan process. The Boer-Mulders function $\left(h_{1}^{\perp}\left(x, k_{T}\right)\right)$ can be derived from the size $(\nu)$ of $\cos 2 \phi$ modulation. SeaQuest finished the second data-taking period in August 2014. Preliminary results of $\bar{d} / \bar{u}$ and $\nu$ are reported.
\end{abstract}

Keywords: Proton Structure; Parton Distribution Function; Light Anti-Quark

PACS numbers: 14.20.Dh, 11.30.Hv, 14.65.Bt

\section{Motivation}

The internal structure of the nucleon is one of the most vital topic in the present hadron physics. The SeaQuest experiment (also known as E906) is being carried out at FNAL to investigate the nucleon structure with the Drell-Yan process. It utilizes several types of target to cover wide physics topics, such as the flavor asymmetry of light anti-quark distributions, the Boer-Mulders function and the nuclear effects such as the parton energy loss in cold nuclei. It also measures the $J / \psi$ production process as another probe.

The distributions of $\bar{u}$ and $\bar{d}$ in the proton were initially assumed to be flavor symmetric, i.e. $\bar{d}=\bar{u}$, because $\bar{u}$ and $\bar{d}$ are created via gluon splittings with the same probability due to their almost same mass. But a large asymmetry was discovered

This is an Open Access article published by World Scientific Publishing Company. It is distributed under the terms of the Creative Commons Attribution 3.0 (CC-BY) License. Further distribution of this work is permitted, provided the original work is properly cited. 
by the $\mathrm{NMC},{ }^{1} \mathrm{NA}^{2} 1^{2}$ and E866/NuSea ${ }^{3}$ experiments. The asymmetry is as large as $\bar{d} / \bar{u}=170 \%$ at $x \sim 0.2$. Many theoretical models have been proposed and compared with the measurements. ${ }^{4}$ SeaQuest aims to measure $\bar{d} / \bar{u}$ at higher $x$ up to 0.45 to reveal the $x$ dependence of $\bar{d} / \bar{u}$.

The Boer-Mulders function $\left(h_{1}^{\perp}\left(x, k_{T}\right)\right)$ is one of the transverse-momentumdependent parton distribution functions. ${ }^{5}$ Experiments of semi-inclusive deepinelastic scattering (SIDIS) discovered that $h_{1}^{\perp}\left(x, k_{T}\right)$ of quarks is non-zero. ${ }^{6}$ Also $h_{1}^{\perp}\left(x, k_{T}\right)$ of anti-quarks was extracted from the Drell-Yan process measured by the E866/NuSea experiment, ${ }^{7,8}$ although the statistical accuracy is quite limited. ${ }^{9,10}$ SeaQuest aims to measure $h_{1}^{\perp}\left(x, k_{T}\right)$ of anti-quarks with a better precision with the Drell-Yan process.

\section{Drell-Yan Process as Probe to Nucleon Structure}

The diagram of the Drell-Yan process is shown in Fig. 1. Its cross section at the leading order of $\alpha$ is written as

$$
\frac{d^{2} \sigma}{d x^{\mathrm{B}} d x^{\mathrm{T}}}=\frac{4 \pi \alpha^{2}}{9 x^{\mathrm{B}} x^{\mathrm{T}}} \frac{1}{s} \sum_{i} e_{i}^{2}\left\{q_{i}^{\mathrm{B}}\left(x^{\mathrm{B}}\right) \bar{q}_{i}^{\mathrm{T}}\left(x^{\mathrm{T}}\right)+\bar{q}_{i}^{\mathrm{B}}\left(x^{\mathrm{B}}\right) q_{i}^{\mathrm{T}}\left(x^{\mathrm{T}}\right)\right\}
$$

where $x$ is the Bjorken scaling variable, $s$ is the square center-of-mass energy of two interacting hadrons, $q_{i}(x)$ is the parton distribution function of a flavor $i$, and the superscripts "B" and "T" denote partons in the beam hadron and the target hadron, respectively. When the process is measured at the forward rapidity as SeaQuest does, the second term $\left(\bar{q}_{i}^{\mathrm{B}}\left(x^{\mathrm{B}}\right) q_{i}^{\mathrm{T}}\left(x^{\mathrm{T}}\right)\right)$ of Eq. 1 is infrequent since $x_{\mathrm{B}} \gg x_{\mathrm{T}}$ (as seen in Fig. 2) and $\bar{q}\left(x^{\mathrm{B}}\right) \sim 0$ at large $x^{\mathrm{B}}$. It means that a quark often comes from the beam with $x^{\mathrm{B}}$ and an anti-quark from the target with $x^{\mathrm{T}}$.

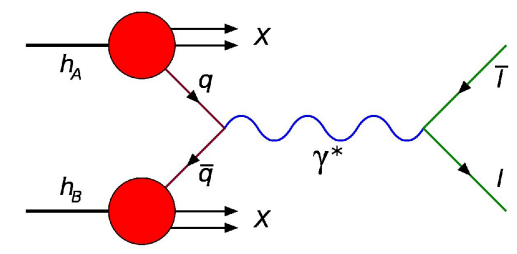

Fig. 1. Diagram of Drell-Yan process, $q+\bar{q} \rightarrow \gamma^{*} \rightarrow \mu^{+}+\mu^{-}$.

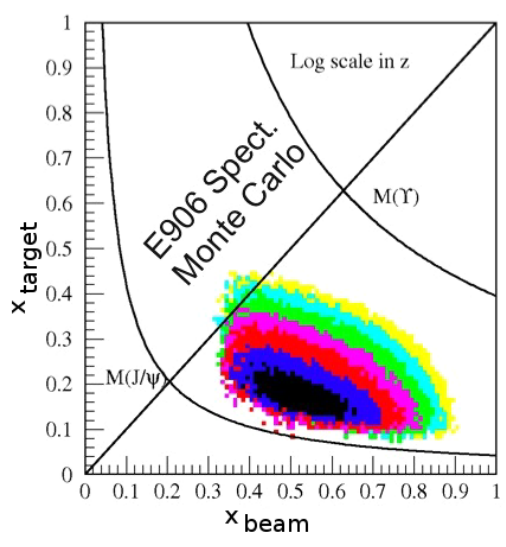

Fig. 2. $x$ of target and beam in DrellYan processes measured by SeaQuest. 
SeaQuest measures the Drell-Yan cross sections in $p+p$ and $p+d$ reactions with liquid hydrogen $\left(\mathrm{LH}_{2}\right)$ and deuterium $\left(\mathrm{LD}_{2}\right)$ targets. The flavor asymmetry is derived from the ratio of the cross sections as

$$
\frac{\sigma_{p+d}}{2 \sigma_{p+p}} \approx \frac{1}{2}\left(1+\frac{\bar{d}}{\bar{u}}\right) .
$$

Most systematic errors of the cross sections are canceled out by taking the ratio.

The angular distribution of Drell-Yan process can be written as ${ }^{11}$

$$
\frac{1}{\sigma} \frac{d \sigma}{d \Omega}=\frac{3}{4 \pi} \frac{1}{\lambda+3}\left(1+\lambda \cos ^{2} \theta+\mu \sin 2 \theta \cos \phi+\frac{\nu}{2} \sin ^{2} \theta \cos 2 \phi\right)
$$

where $\phi$ and $\theta$ are the angle of $\mu^{+}$in the dimuon rest frame called the Collins-Soper frame. The parameter $\nu$ quantifies the size of $\cos 2 \phi$ modulation, and is proportional to the Boer-Mulders functions of two interacting quarks; $\nu \propto h_{1}^{\perp}\left(x, k_{T}\right) \times h_{1}^{\perp}\left(x, k_{T}\right)$. Under the experimental condition of SeaQuest, one $h_{1}^{\perp}\left(x, k_{T}\right)$ is of quark at $x \sim 0.5$ and the other $h_{1}^{\perp}\left(x, k_{T}\right)$ is of anti-quark at $x \sim 0.3$.

\section{Setup and Schedule}

SeaQuest is being carried out at FNAL. It uses the proton beam extracted from the FNAL Main Injector. The beam energy is $120 \mathrm{GeV}$, which corresponds to $\sqrt{s}=15$ $\mathrm{GeV}$. The beam is slow-extracted for 5 seconds every 60 seconds. Typically 40,000 protons are bunched with a length of about 1 ns and an interval of 19 ns (i.e. 53 $\mathrm{MHz}$ ). Per duty cycle (60 seconds), $10^{13}$ protons are injected into a target.

Figure 3 is a schematic drawing of the SeaQuest spectrometer.

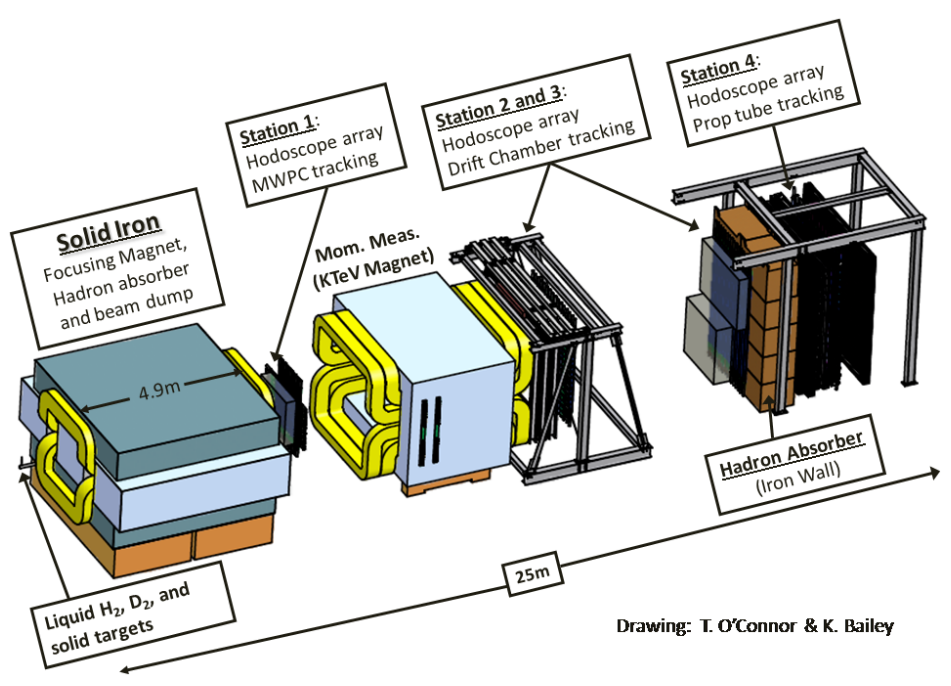

Fig. 3. Schematic drawing of E906/SeaQuest spectrometer. 
The $\mathrm{LH}_{2}$ and $\mathrm{LD}_{2}$ targets are used for the measurement of Drell-Yan processes in $p+p$ and $p+d$ reactions. Carbon, iron and tungsten are also used for measurements of the nuclear effects. One target in rotation per spill is irradiated with the beam.

The first magnet behind the target (called "FMag") is to focus muons of DrellYan processes on the spectrometer acceptance. Also it is filled with solid iron to absorb the beam and its secondary particles.

Muons are detected with four groups of detectors, called stations. The trajectory of muons is reconstructed with drift chambers at Stations 1, 2 and 3. The second magnet (called "KMag") is placed between Stations 1 and 2 to measure muon momenta. Station 4 consists of proportional tubes and a hadron absorber. A particle is identified as muon if it penetrates the hadron absorber and hit the proportional tubes at Station 4. Every station is equipped with a hodoscope array for data acquisition trigger. High-mass muon pairs are selectively triggered.

Regarding the schedule of SeaQuest, the proton beam was provided in three periods up to now. The first one was a short run to commission the beam and the spectrometer in 2012. The second and third ones were to accumulate physics events. The third one is on-going now. The data taking will finish in 2016 to have $10^{19}$ protons delivered.

\section{Results}

The second data taking (called "Run 2") was done for 10 months up to August 2014. A part of Run-2 data has been analyzed. It is about $1 / 3$ of the statistics in Run 2 and about 1/20 of the anticipated statistics in all the data takings.

Figure 4 shows the invariant mass of dimuons measured with all targets $\left(\mathrm{LH}_{2}\right.$, $\mathrm{LD}_{2}, \mathrm{C}, \mathrm{Fe}$ and $\left.\mathrm{W}\right)$. The distribution is fitted with four components; Drell-Yan

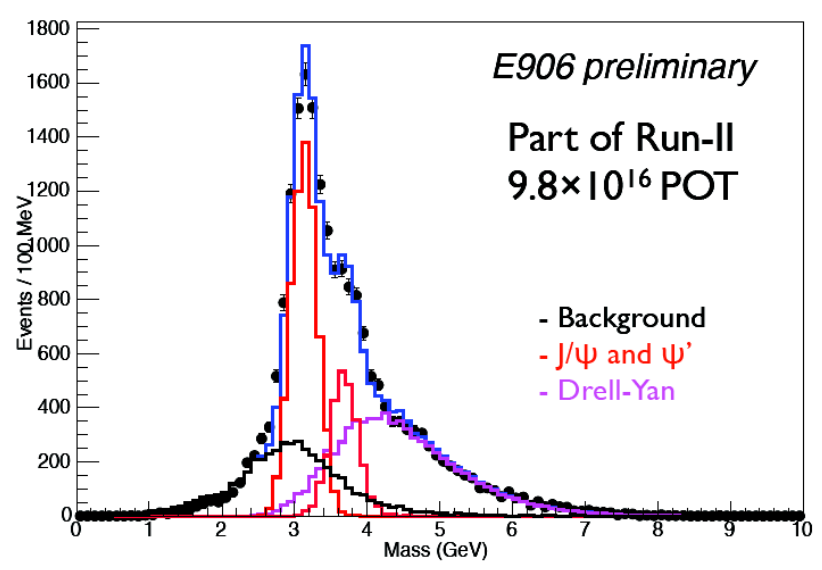

Fig. 4. Invariant mass of dimuons. The black points are the measured result with all the targets. The red, black and purple solid lines are the components of $J / \psi, \psi^{\prime}$, Drell-Yan and background events. They are fitted to the black points, and the blue solid line is the sum of the fitted components. 
events, $J / \psi$ events, $\psi^{\prime}$ events, and random backgrounds. The distribution shapes of the Drell-Yan, $J / \psi$ and $\psi^{\prime}$ events were estimated with simulation. That of the random backgrounds was estimated with real data.

The measured distribution has been reasonably well fitted. It suggests that the resolution and the acceptance of the spectrometer has been understood. The mass resolution is $0.18 \mathrm{GeV}$ at $3 \mathrm{GeV}$, as evaluated with the width of $J / \psi$ peak. It satisfies the design requirement for the spectrometer, and enables us to separate the $J / \psi$ and $\psi^{\prime}$ peaks. The Drell-Yan process dominates at mass $>4.2 \mathrm{GeV}$.

Drell-Yan events were selected with a cut of mass $>4.2 \mathrm{GeV}$, and counted on each target. The yield on each target was normalized with the number of beam protons on the target. The ratio of normalized yields of two targets is equal to the cross-section ratio of two targets, where all efficiencies in measurement are assumed to be canceled out.

The flavor asymmetry $(\bar{d} / \bar{u})$ is derived from the measured $\sigma_{p+d} / \sigma_{p+p}$ using Eq. 2 . Figure 5 shows the statistical accuracy of the analyzed data on $\bar{d} / \bar{u}$. The magnitude and systematic error of $\bar{d} / \bar{u}$ are being evaluated. The accuracy will be better by a factor of $4.5(=\sqrt{20})$ once the full statistics are accumulated and analyzed. The anticipated accuracy shown in Fig. 5 should be achievable. The measurement efficiency is being improved also.

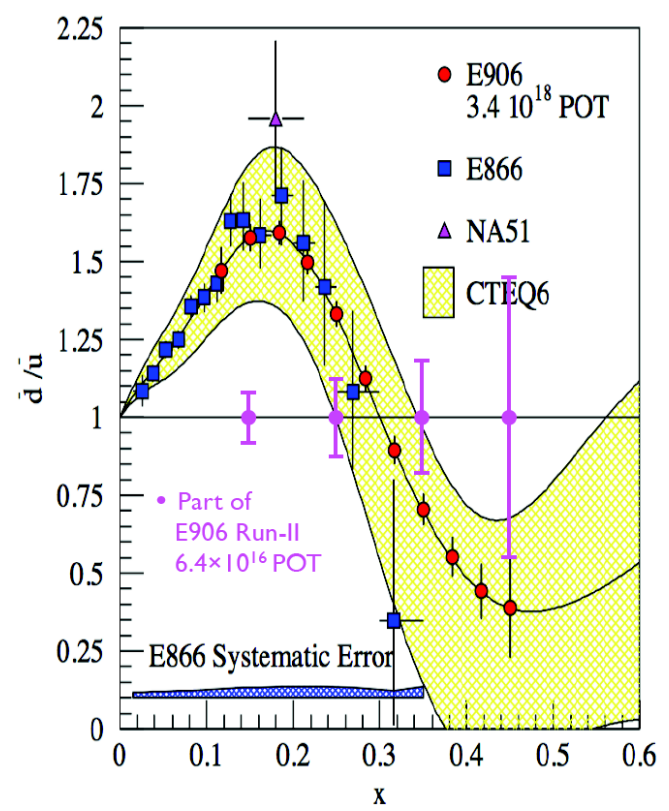

Fig. 5. Flavor asymmetry $(\bar{d} / \bar{u})$ as a function of $x$. The statistical accuracy of the SeaQuest analyzed data is shown as the size of purple vertical bars around $\bar{d} / \bar{u}=1$. The vertical bars on the red circles are the anticipated statistical accuracy with the full statistics of SeaQuest. The pink triangle is the result of CERN NA51, and the blue squares are of FNAL E866/NuSea. The solid line with the yellow band is the CTEQ6 fit result. 


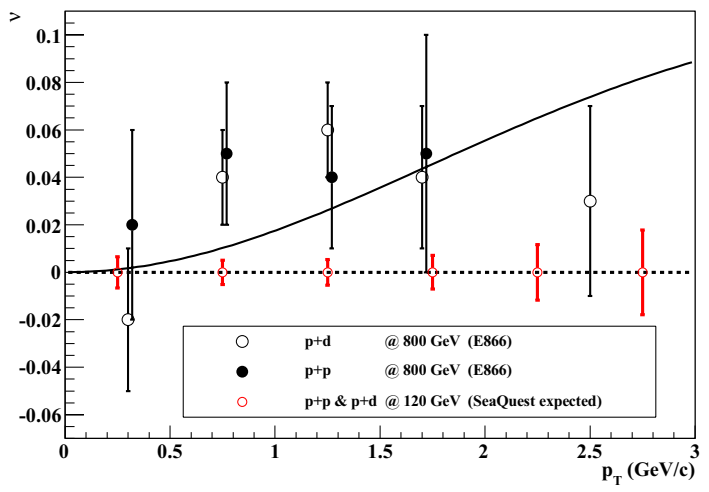

Fig. 6. The size $(\nu)$ of the $\cos (2 \phi)$ modulation of Drell-Yan process as a function of the dimuon transverse momentum $\left(p_{T}\right)$. The anticipated accuracy of the SeaQuest full statistics is shown as the size of red vertical bars around $\nu=0$. The open and filled circles are the result of E866/NuSea.

The size $(\nu)$ of the $\cos (2 \phi)$ modulation is measured to extract the Boer-Mulders function. Analyses of the Run-2 data are ongoing. The anticipated accuracy with the SeaQuest full statistics has been evaluated with simulation, and is shown in Fig. 6. It demonstrates that SeaQuest measures $\nu$ with the better accuracy.

\section{Conclusions}

SeaQuest is the running experiment at FNAL to investigate the nucleon structure with the Drell-Yan process. It finished the second data taking (Run 2) in August 2014. About $1 / 3$ of Run-2 data has been analyzed. The preliminary result of the flavor asymmetry $(\bar{d} / \bar{u})$ with the part of Run-2 data was presented. The anticipated accuracy of the Drell-Yan angular distribution was evaluated, where the Boer-Mulders function $\left(h_{1}^{\perp}\left(x, k_{T}\right)\right)$ can be derived from the size $(\nu)$ of the $\cos (2 \phi)$ modulation. Twenty times more statistics are anticipated after the data taking is completed in 2016.

\section{References}

1. K. Gottfried, Phys. Rev. Lett. 18, p. 1174 (1967).

2. A. Baldit et al., Phys. Lett. B332, 244 (1994).

3. R. S. Towell et al., Phys. Rev. D64, p. 052002 (2001).

4. J. C. Peng et al., Phys. Rev. D58, p. 092004 (1998).

5. D. Boer and P. Mulders, Phys.Rev. D57, 5780 (1998).

6. V. Barone, S. Melis and A. Prokudin, Phys.Rev. D81, p. 114026 (2010).

7. L. Zhu et al., Phys.Rev.Lett. 99, p. 082301 (2007).

8. L. Zhu et al., Phys.Rev.Lett. 102, p. 182001 (2009).

9. Z. Lu and I. Schmidt, Phys.Rev. D81, p. 034023 (2010).

10. V. Barone, S. Melis and A. Prokudin, Phys.Rev. D82, p. 114025 (2010).

11. C. Lam and W.-K. Tung, Phys.Rev. D18, p. 2447 (1978). 